Meta

Journal des tradlucteurs

Translators' Journal

Tallers de Letras (1990) : Revista del Instituto de Letras de la Pontificia Universidad Católica de Chile, no18, 179 p.

\title{
Jean-Claude Gémar
}

Volume 37, numéro 2, juin 1992

URI : https://id.erudit.org/iderudit/002858ar

DOI : https://doi.org/10.7202/002858ar

Aller au sommaire du numéro

\section{Éditeur(s)}

Les Presses de l'Université de Montréal

\section{ISSN}

0026-0452 (imprimé)

1492-1421 (numérique)

Découvrir la revue

Citer ce compte rendu

Gémar, J.-C. (1992). Compte rendu de [Tallers de Letras (1990) : Revista del Instituto de Letras de la Pontificia Universidad Católica de Chile, no18, 179 p.]

Meta, 37(2), 354-355. https://doi.org/10.7202/002858ar services d'Érudit (y compris la reproduction) est assujettie à sa politique d'utilisation que vous pouvez consulter en ligne.

https://apropos.erudit.org/fr/usagers/politique-dutilisation/ 
Taller de Letras (1990) : Revista del Instituto de Letras de la Pontificia Universidad Católica de Chile, no 18, 179 p.

Chaque année, l'Institut des lettres de 1'Université catholique du Chili, à Santiago, publie un numéro spécial de sa revue consacré à un thème, à un auteur ou à une discipline. Il peut porter sur la poésie, la littérature, une discipline ou une œuvre en particulier. C'est ainsi que le prochain numéro, annoncé par les auteurs, traitera de la recherche en traduction. Le numéro 18 présente en douze articles (dont 8 en espagnol, 3 en français et 1 en allemand) et deux volets l'activité du traducteur envisagée d'abord sous son aspect professionnel, puis d'un point de vue universitaire.

L'Amérique latine suit avec grand intérêt l'évolution de la traduction, de ses techniques et de son enseignement. En témoignent de nombreux travaux et écrits dans la plupart des pays latino-américains. Quel traducteur n'a entendu parler d'Octavio Paz et de ses réflexions, aussi brillantes que pertinentes, sur la traduction publiée, entre autres, dans la revue de 1'Unesco? L'équipe qui a réalisé le numéro spécial de Meta sur la traduction dans le monde hispano-lusophone (35-3), avec la Profesora Ileana Cabrera à sa tête, est aussi celle qui dirige Taller de Letras et a réalisé le numéro 18. Celui-ci comprend en fait 3 parties, dont les deux premières (jusqu'à la page 120) sont consacrées à l'activité de traduction déjà signalée. La troisième expose les résultats d'une enquête portant sur la formation du traducteur et l'état de la recherche en traduction dans les universités de trois grands pays d'Amérique latine (Argentine, Chili et Pérou), suivis d'annexes, les questionnaires (vierges) adressés aux responsables et aux chercheurs des universités de ces pays. Les résultats présentés sont censés refléter l'état actuel de la formation et de la recherche universitaires en traduction dans cette région de l'Amérique latine.

Dans le premier volet de l'activité de traduction, le «professionnel», cinq articles ou brefs essais - pour 3 d'entre eux - mettent (René Chapero, p. 9) le traducteur en scène 
et les exigences des organisations internationales en exergue, esquissent l'environnement du traducteur de demain (André Clas, p. 15), préviennent contre les dangers que présente l'informatique en traductologie (Christine Durieux, p. 25), exposent (Bárbara Trosko, p. 33), dans une brève note, le problème posé par la traduction en espagnol du titre du roman d'Anthony Burgess, Orange mécanique, et du temps (le passé ou le futur?), évoquent (Alicia Morel, p. 37), enfin, en une courte «recette», la «cuisine littéraire» cachée de Katherine Mansfield. Le lecteur trouvera donc à boire et à manger dans une juxtaposition - hétéroclite et inégale - de textes qui reflète une conception peu orthodoxe de la profession de traducteur.

Le deuxième volet, qui porte sur l'activité «universitaire», en fait la traductologie, comprend sept articles, dont trois tournent autour de l'enseignement de la traduction. Dans le premier (p. 41), Ileana Cabrera et Patricia Hörmann abordent un sujet délicat, l'évolution des «hypothèses théoriques» (supuestos teóricos). Elles analysent notamment les deux étapes «scientifiques» de cette évolution: la descriptive et l'explicative, en s'appuyant sur quelques travaux, dont ceux de Juan Luis Vives, Vinay et Darbelnet, Nida, Newmark, Seleskovitch et Frank Königs. Cecilia Beuchat et Carolina Valdivieso (p. 55) traitent des difficultés que présente la traduction de la littérature enfantine, en raison particulièrement des transpositions obligées des dénotations culturelles et, peut-être encore davantage, des aspects culturels des connotations, abstractions et interprétations. Karin Riedeman (p. 65) aborde la traduction comme phénomène pragmatique - au sens $d^{\prime}$ 'intention particulière» - et en tire des conclusions pédagogiques. María Isabel Diéguez (p. 75) envisage l'enseignement de la traduction du point de vue psycholinguistique et en présente une application pratique. Jean-Claude Gémar (p. 83) traite des apports méthodologiques de la jurilinguistique en traduction et terminologie spécialisées, mais également générales. Un collectif de professeurs chiliens (p. 93) traite de la question de l'emprunt linguistique comme procédé de traduction. Frank Königs (p. 103), enfin, dans le dernier article de la série, pose le problème de la théorie face à la pratique, analyse et explicite certains aspects théoriques de la traduction, qu'il subdivise, à partir de la métathéorie, en trois niveaux ou ordres (Ordnung) hiérarchisés et présente selon le modèle proposé.

L'intérêt pour la recherche en traduction et l'enseignement de cette discipline gagne partout du terrain. Les pays du Sud en ressentent le besoin et font de grands efforts pour suivre l'évolution des choses, tenter de réduire l'écart. Et cela non seulement dans le secteur économique et industriel, mais également dans le domaine de l'intellect comme, par exemple, celui de la formation universitaire des futures générations de traducteurs, laquelle repose de plus en plus sur une base méthodologique rigoureuse et bien établie. La croissance et la diversification des échanges internationaux obligent à repenser les termes du dialogue inter- (et intra) universitaire dans le sens d'une plus grande ouverture sur un monde multipolaire, multilingue et multiculturel où le traducteur est plus que jamais appelé à jouer, qu'il le veuille ou non, le rôle de médiateur qui lui est traditionnellement reconnu. Il faut encourager ces efforts, au risque de les voir emprunter des sentiers battus ou des voies sans issue pour reconnaître un parcours déjà tracé. La porte de la connaissance est étroite, mais toujours ouverte. 